\title{
Dried and Redispersible Cellulose Nanocrystal Pickering Emulsions
}

\author{
Zhen Hu, Heera S. Marway, Hesham Kasem, Robert Pelton, and Emily D. Cranston* \\ Department of Chemical Engineering, McMaster University, 1280 Main Street West, Hamilton, \\ Ontario L8S 4L7, Canada \\ * Corresponding Author \\ E-mail: ecranst@mcmaster.ca.
}

Materials. Methyl cellulose (MC, $40 \mathrm{kDa}$, degree of substitution of 1.6-1.9), 2-hydroxyethyl cellulose (HEC, $90 \mathrm{kDa}$, molar substitution of 2.5), tannic acid (TA), corn oil, Nile red, and 2-[4(2-hydroxyethyl)piperazin-1-yl]ethanesulfonic acid (HEPES buffer) were all purchased from Sigma-Aldrich. All water used was deionized and further purified with a Barnstead Nanopure Diamond system (Thermo Scientific).

Preparation of Cellulose Nanocrystals. Cellulose nanocrystals (CNCs) were prepared by sulfuric acid hydrolysis of cotton from cotton filter aid (Whatman ashless filter aid, GE Healthcare Canada), as described previously. ${ }^{1}$ Briefly, $40 \mathrm{~g}$ of the filter aid was treated with 700 $\mathrm{mL}$ of $64 \mathrm{wt} \%$ sulfuric acid (Fischer Scientific) for $45 \mathrm{~min}$ at $45{ }^{\circ} \mathrm{C}$ with constant stirring (mechanical stirrer) in a water bath. The hydrolysis reaction was quenched with cold water and acid/degraded sugars were removed by centrifugation and extensive dialysis against purified water. The surface charge density of sulfate half-ester groups on CNCs was determined by conductometric titration to be $0.33 \pm 0.02 \mathrm{e} / \mathrm{nm}^{2}$, with average crystal dimensions of $128 \times 7 \mathrm{~nm}$ from transmission electron microscopy (TEM) image analysis.

Preparation of Emulsions. Emulsions of $10 \mathrm{~mL}$ containing $2 \mathrm{~mL}$ oil (dodecane or corn oil) and $8 \mathrm{~mL}$ aqueous dispersions with $20 \mathrm{mM}$ HEPES buffer $(\mathrm{pH} 8)$ were obtained using a probe sonicator (Sonifier 450, Branson Ultrasonics), in an ice bath for $1 \mathrm{~min}$ at an intensity level 6 and 
$50 \%$ pulses. For the emulsions stabilized with CNCs and polymer or CNCs and tannic acid, water-soluble components were dissolved in aqueous phase first and then mixed with oil before emulsification. For the emulsions stabilized with CNCs, polymer, and tannic acid, tannic acid was added later to the emulsions stabilized by $\mathrm{CNCs}$ and polymer and mixed for $6 \mathrm{~h}$ before analysis.

Confocal Laser Scanning Microscopy (CLSM). CLSM was use to investigate the microstructure of the emulsions; the oil phase was stained with Nile red $(492 / 520 \mathrm{~nm})$, as described previously. ${ }^{2}$ Images were obtained with a Zeiss LSM 510 Meta on an Axiovert 200 M microscope (Zeiss, Gottingen, Germany). For high resolution images, a $63 \times$ water-immersion laser technique was used.

Scanning Electron Microscopy (SEM). The oil powders were metallized with $5 \mathrm{~nm} \mathrm{Pt}$ and visualized using a JEOL 7000F SEM (JEOL Ltd., Japan). Micrographs were taken at $8 \mathrm{~mm}$ working distance with $5 \mathrm{kV}$ acceleration voltage.

Malvern Mastersizer. A Malvern Mastersizer 2000G instrument with a HeNe laser operating at $633 \mathrm{~nm}$ was used to measure the emulsion droplet size. The mean droplet diameter was taken to be the volume mean diameter $\left(\mathrm{D}_{4 / 3}\right)$ from 3 replicate measurements. It is mathematically expressed as $D_{4 / 3}=\Sigma D_{i}^{4} N_{i} / \Sigma D_{i}^{3} N_{i}$, where $D_{i}$ is the droplet diameter for droplet fraction $\mathrm{i}$, and $\mathrm{N}_{\mathrm{i}}$ is the number of droplets with size $D_{i}$. The "span" was reported as a measurement of the emulsion droplet diameter size distribution and calculated using span $=(\mathrm{D}(0.9)-\mathrm{D}(0.1)) / \mathrm{D}(0.5)$, where $\mathrm{D}(0.9)$ is the droplet diameter for which $90 \%$ of the droplets are below this size, $\mathrm{D}(0.5)$ is the droplet diameter for which $50 \%$ of the droplets are smaller than this size, and $\mathrm{D}(0.1)$ is the diameter for which only $10 \%$ of the droplets lie below this size. 
Transmission Electron Microscopy (TEM). A single drop of MC-TA or HEC-TA colloidal complex suspension was dropped on a Formvar-coated copper TEM grid and dried for $4 \mathrm{~h}$. TEM images were acquired using a JEOL 1200 EX TEMSCAN microscope operating at $80 \mathrm{kV}$.

Quartz Crystal Microbalance with Dissipation Monitoring (QCM-D). CNC-coated sensors were prepared using an approach that was previously used in similar work. ${ }^{1} \mathrm{MC}$ adsorption on CNC surfaces was measured with an E4 QCM-D instrument from Q-Sense AB (Sweden) where the third, fifth, and seventh overtones were recorded. An aqueous solution of HEPES buffer was injected at a constant flow rate of $150 \mu \mathrm{L} /$ min until the baseline frequency shift was less than 0.5 Hz over $10 \mathrm{~min}$. Then $1 \mathrm{~g} / \mathrm{L}$ MC in HEPES buffer was injected at $150 \mu \mathrm{L} / \mathrm{min}$ until the baseline was again stable, typically after $60 \mathrm{~min}$. The sensor was rinsed with HEPES buffer at $150 \mu \mathrm{L} /$ min until the baseline was stable, typically after $5 \mathrm{~min}$. The same adsorption-rinsing procedure was repeated for $2 \mathrm{~g} / \mathrm{L}$ TA in HEPES buffer.
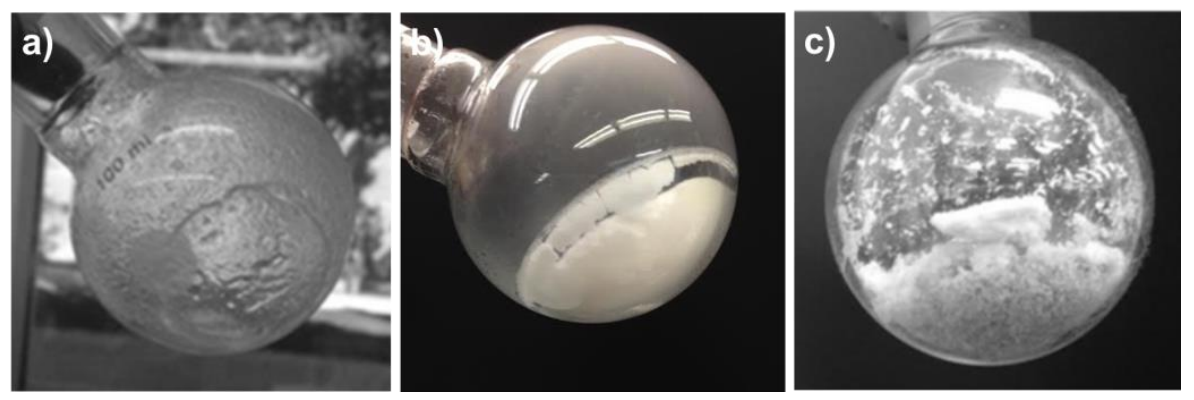

Figure S1. Photographs of emulsions (20 vol \% corn oil) after freeze-drying, stabilized by (a): CNCs $(0.25$ wt $\%)$ - these emulsions could not be freeze-dried without emulsion breaking; (b): CNCs $(0.25 \mathrm{wt} \%)$ and MC $(0.75 \mathrm{wt} \%)$ - these emulsions could be freeze-dried but were not redispersible as shown in Fig S2 and S3 where the emulsion phase separates and droplet coalescence is evident; and (c): $\mathrm{CNCs}(0.25 \mathrm{wt} \%), \mathrm{MC}(0.25 \mathrm{wt} \%)$, and TA $(0.5 \mathrm{wt} \%)$ - these emulsions could be freeze-dried and redispersed. 


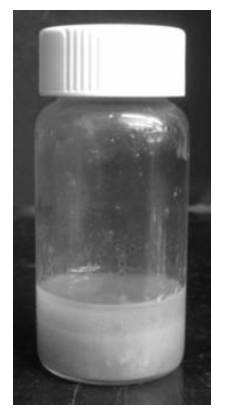

Figure S2. Photograph of freeze-dried emulsions (20 vol \% corn oil) stabilized by CNCs $(0.25$ wt \%) and $\mathrm{MC}(0.75 \mathrm{wt} \%)$ upon re-hydration showing phase separation of the oil and water phases.
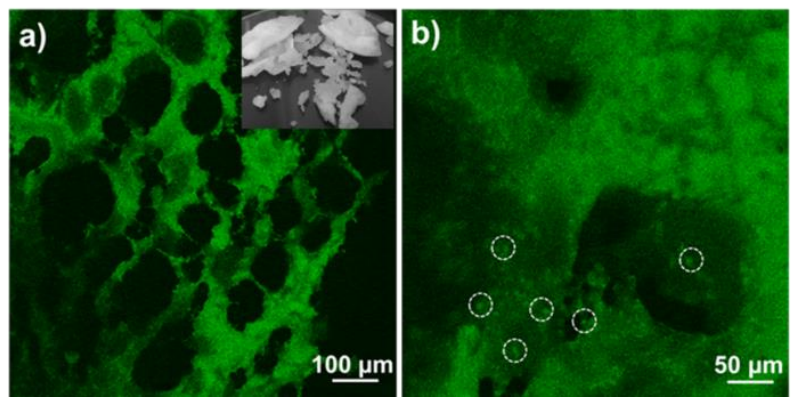

Figure S3. Confocal laser scanning microscopy (CLSM) images of freeze-dried emulsions (20 vol \% corn oil) stabilized by CNCs $(0.25 \mathrm{wt} \%)$ and $\mathrm{MC}(0.75 \mathrm{wt} \%)$ at (a) low and (b) high magnification. Inset picture is a photograph of the freeze-dried emulsion.
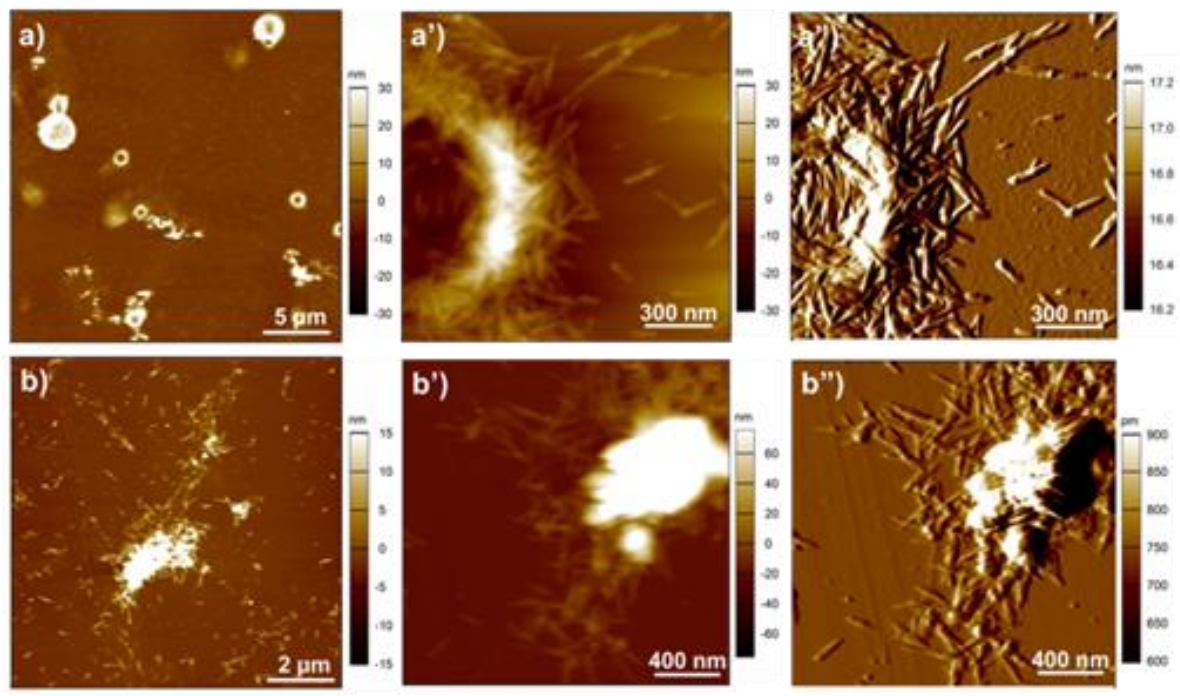

Figure S4. Atomic force microscopy (AFM) images: dodecane-in-water emulsions (20 vol \% dodecane) stabilized by CNCs $(0.25 \mathrm{wt} \%)$ and $\mathrm{MC}(0.75 \mathrm{wt} \%)$, (a) and (a') height images, (a'') amplitude image; dodecane-in-water emulsions (20 vol \% dodecane ) stabilized by CNCs $(0.25$ wt \%), MC (0.25 wt \%), and TA (0.5 wt \%), (b) and (b') height images, (b'’) amplitude image. 
To determine the role of TA in producing redispersible oil powders, CNC-MC and CNC-MC-TA emulsions (dodecane-in-water) were air-dried on silica wafers and imaged by AFM. As shown in Figure S4a, dried CNC-MC emulsion formed coffee ring structures upon drying. The rodlike CNCs can be viewed at higher magnification in Figure S4a' and S4a". On the other hand, no clear coffee ring structures were observed with the CNC-MC-TA sample, which might be due to the change of stickiness of MC-coated CNC particles after addition of TA. ${ }^{3}$ Interestingly, some round-shaped nanoparticles can be observed in Figure S4b' and S4b", likely due to the formation of colloidal complexes between MC and TA, as evidenced by QCM-D results shown in Figure 2. ${ }^{4,5}$ Our previous work suggests that the oil-water interface is covered by both CNC particles coated with MC or HEC and free MC or HEC chains not bound to cellulose. ${ }^{6}$ The formation of colloidal complexes between MC and TA leads to insoluble complexes of the polymers and TA at the oil-water interface which provides additional stabilization capability. ${ }^{7}$
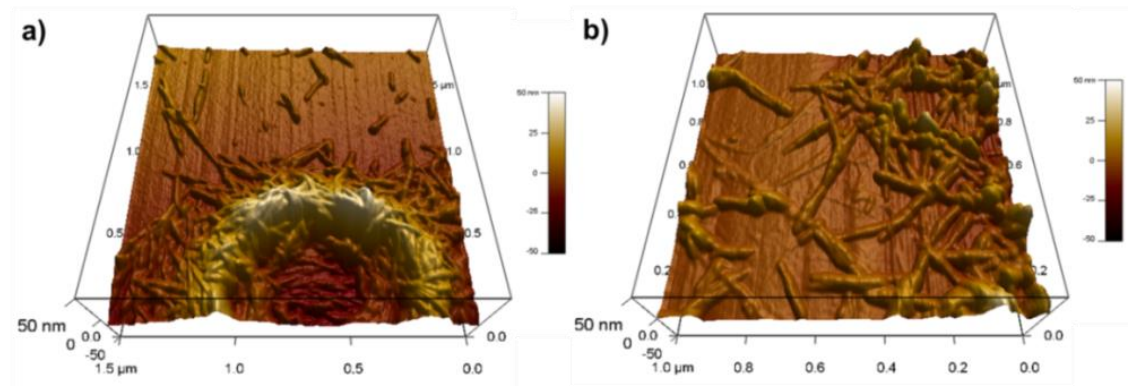

Figure S5. Atomic force microscopy (AFM) 3D height images of dodecane-in-water emulsions (20 vol \% dodecane) stabilized by (a) CNCs (0.25 wt \%) and MC (0.75 wt \%); (b) CNCs (0.25 wt \%), MC (0.25 wt \%), and TA $(0.5 \mathrm{wt} \%)$.
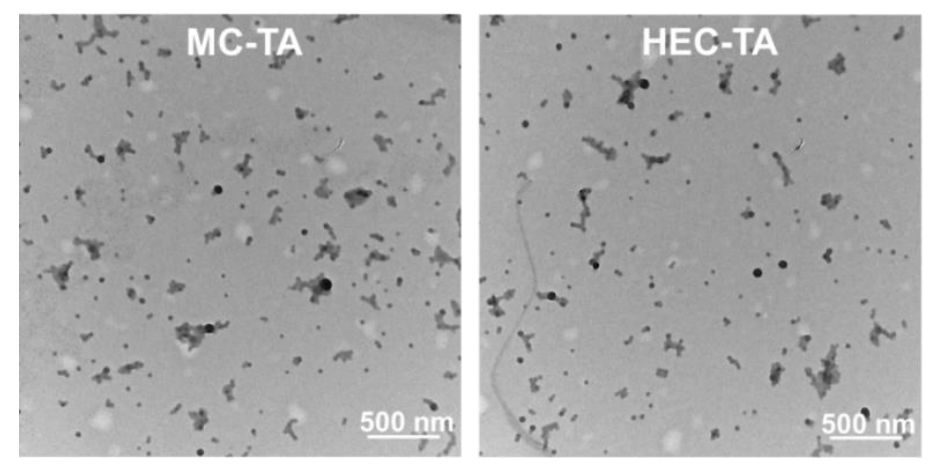

Figure S6. Transmission electron microscopy (TEM) images of colloidal complexes prepared by mixing (left) MC (0.1 wt \%) and TA (0.1 wt \%) and (right) HEC (0.1 wt \%) and TA (0.1 wt \%).

Average particle size of MC-TA and HEC-TA complexes is determined by using dynamic light scattering to be $49.7 \pm 3.5 \mathrm{~nm}$ and $53.1 \pm 4.2 \mathrm{~nm}$, respectively. 


\section{References:}

(1) Hu, Z.; Cranston, E. D.; Ng, R.; Pelton, R. Langmuir 2014, 30, 2684-2692.

(2) Hu, Z.; Ballinger, S.; Pelton, R.; Cranston, E. D. Journal of Colloid and Interface Science 2015, 439, 139-148.

(3) Anyfantakis, M.; Baigl, D. Angewandte Chemie International Edition 2014, 53, 14077 14081.

(4) Patel, A. R.; Seijen ten-Hoorn, J.; Hazekamp, J.; Blijdenstein, T. B. J.; Velikov, K. P. Soft Matter 2013, 9, 1428-1436.

(5) Patel, A. R.; Seijen-ten-Hoorn, J.; Velikov, K. P. Journal of Colloid and Interface Science 2011, 364, 317-323.

(6) Hu, Z.; Patten, T.; Pelton, R.; Cranston, E. D. ACS Sustainable Chemistry \& Engineering 2015, 3, 1023-1031.

(7) Driver, K.; Baco, S.; Khutoryanskiy, V. V. European Polymer Journal 2013, 49, 42494256. 